\title{
RFID Authentication of Academic Transcripts: In the Context of a RFID Rationale and Deployment Methodology
}

\author{
Eugene Lutton, Brian Regan and Geoff Skinner \\ Faculty of Science and Information Technology \\ The University of Newcastle \\ Australia \\ eugene.lutton; brian.regan; geoff.skinner @newcastle.edu.au
}

\begin{abstract}
The integrity and authentication of an academic transcript is vital to the prestige of an educational organisation as well as its graduates. It is important to the organisation issuing the transcript and any future educational organisation that uses the transcript to ascertain the merit of a proposed enrolment or academic employment. The business case for this research is undertaken within the boundaries of a radio frequency identification rationale and deployment methodology. It is proposed to utilise radio frequency identification technology to enhance the current process of creating and validating academic transcripts. This paper will investigate the business case for radio frequency identification technology and how this correlates with the business environment which is the first phase of the methodology.
\end{abstract}

Keywords-rfid methodology; academic transcripts; rfid deployment; radio frequency identification;

\section{INTRODUCTION}

This paper investigates the business case of using radio frequency identification (RFID) to improve the integrity and authentication of an academic transcript at The University of Newcastle, Australia. The business case for this possible enhancement will be analysed within the boundaries of a RFID Rationale and Deployment Methodology previously developed by the author [3],[4]. This methodology has three phases, namely the business, infrastructure and deployment environments. This current research will analyse the business case within the context of the first phase of the methodology.

The structure of this paper is as follows; Section II discusses the background of this research, Section III examines the current research problem, Section IV outlines the solution for the enhanced authentication of an academic transcript, Section V discusses the limitations, future research and finishes with the conclusion.

\section{RESEARCH BACKGROUND}

After extensive research, and to the best of our knowledge there has been minimal literature on RFID enabled academic transcripts. India's University of Hyderabad has embedded RFID tags within their paper diplomas [1]. They have utilised passive tags with 4 Kilobytes of memory operating at the $13.56 \mathrm{MHz}$ spectrum. The business case was to reduce fraudulent practises with their diplomas. Their reputation for computer science was an important motivator for improving the government directive of adding a photo identification to diplomas [1]. At the time of writing this paper, Universities in the United Arab Emirates (UAE) [2] are issuing academic diplomas with passive RFID tags attached. The spectrum is the same as utilised in India [1]. The motivation was to ensure validity of a diploma and to improve student registration. In both of the previous cases student data will be stored in the tags memory. This following section will present the RFID Rationale and Deployment Methodology and how the current research fits within this methodology.

\section{A. RFID Rationale Deployment Methodology}

This methodology was developed to assist an organisation with their decision making and understanding of a possible deployment of a RFID system. The RFID Rationale and Deployment Methodology is divided into three distinct phases for a RFID deployment.

- Phase 1: Business Environment,

- Phase 2: Infrastructure Environment,

- Phase 3: Deployment Environment.

The business environment examines the current status of the organisation and how their discretionary behaviour is modified by the instigating environment. It is also important to understand the motivation to deploy a RFID system, and what metrics are conducive to continue with the possible deployment. The Infrastructure Environment examines the physics and technical needs of RFID, and how this must be included in the objectives and processes of the business case. The deployment environment investigates and documents the outcomes from operating a use case process once it is enabled with RFID technology. For a more in-depth clarification and explanation of the phases and their relationships refer to [4].

The business environment has four processes as shown in Figure 1 that are followed depending on the current economic, competitive, political or legal circumstances of an organisation. The first step in the business environment is the instigating environment. One of the crucial elements in this process is the clarification of the rationale, for investigating RFID technology. There are likely two major factors that 
initiate this action. This is depicted in Figure 1 by the two paths leading from the instigating environment.

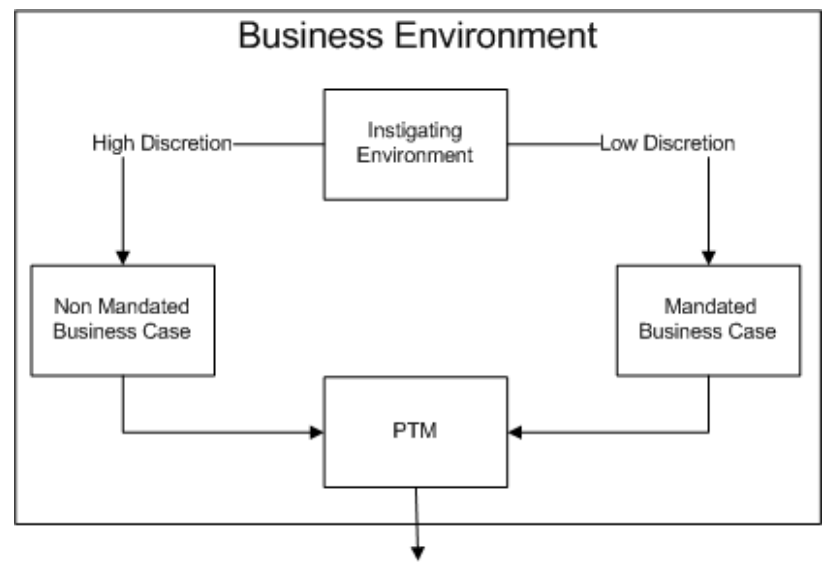

Figure 1. Business Environment

One of these factors is a mandate from an external organisation such as Wal-Mart or The United States Department of Defence (DOD) [5]. In this scenario the path has a tag of low discretion as it flows to a mandated business case. The high discretion path from the instigating environment is followed, if the organisation is examining RFID from an investigative background. In this case the tag shows a high discretion, compared to low discretion leading from a mandated approach. The effect of discretionary behaviour [6] demonstrated that the amount of discretion impacts on the decision making process in a business environment. This analysis of the impact of discretion needs to consider the views of sense-making [7] and satisficing [8] in relation to the organisational decision making process, and the available information and prevailing circumstances.

In the current research the instigating environment for the possible enhancement of an academic transcript has not come from a mandated directive as in the case of [1],[2]. The objectives of the business case are to improve the validity and integrity of academic transcripts. The University of Newcastle is examining this technology from an investigative perspective. This gives them high discretion, and the pathways flows to the non mandated business case, in the business environment as shown in Figure 1. This step in the business environment must analyse the business case and how it fits within the objectives of the organisation.

Due to space limitations summary of the results from examining the business case will not be presented. Once an understanding of the business case is completed, the next process in the business environment is to ascertain the phase transitional motivators (PTM). It is crucial to understand the metrics, or in this case, the PTM that will assist the organisation with their decision to continue the investigation beyond the business environment. In essence, the PTM process looks at the weighting and feasibility given to a PTM, and what is the outcome once analysis has been taken and what action is taken in regards to a PTM. The action relates to the continuance or exit from the analysis of a RFID deployment. Further explanation of the PTM can be found in [3],[4].

The academic transcript business case PTMs include some of the following:

- increase confidence in transcript,

- increase creditability of the transcript,

- gain competitive advantage,

- produce robust administrative processes,

- cost considerations

- RFID reliability

- interaction with existing information systems

- privacy and legal considerations

In this case the interaction with existing information systems, and privacy and legal considerations are important motivators for continuing the investigation. In terms of the methodology, this relates to the infrastructure phase, and associated technical examinations, such as software interaction, data requirements and physical characteristics of the transcript with an embedded RFID tag. The research problem will now be discussed.

\section{Research PRoblem}

This section will discuss the research problem in relation to the current process of creating an academic transcript for a graduation ceremony or a random request by a student for a copy of their results. This is followed by Section III-B with a brief outline of RFID technology in the context of this study.

\section{A. Academic Transcript}

Academic transcripts are an integral representation of a student's educational outcomes. In the context of this research the academic transcript will be related to the one currently utilised by The University of Newcastle Australia. The document contains information that relates to the university, student name and identification number, course title, course grades and results, year attempted, degree and awards. The transcript contains the complete record (history) of all courses a student has been enrolled in, during their time at the university. This may include academic studies completed at an undergraduate or post graduate level. The following paragraphs will describe the current process that is undertaken to prepare and print a transcript that is required for a graduation ceremony, or requested by an individual student

The university sources preprinted paper from an external organisation. To improve the integrity of the transcript the paper has university icons, unique patterns, and various shades of blue printed to the edge of the paper. This format of the paper is to increase the level of difficulty to reproduce or replicate a transcript. 
At the time of writing there are two actions that can initiate the printing of a transcript.

- Student requesting a copy,

- Producing the requirements for a graduation ceremony.

In the first example the student will make a request by phone, email or in person to the relevant department. This request will be recorded and then the student will be notified when the process has been completed. At some time in this process the student must show their student card or relevant information to retrieve the transcript. In the case of a graduation ceremony the process is similar except the print request is related to the students that have accomplished the requirements to participate in the ceremony. In both examples the corporate information system is queried for the academic results of a particular student. This request creates a pdf file that is sent to the designated printer.

Generally this printing process is not completed in real time outside the graduation ceremony time frame. During preparation for graduation ceremonies the printing will be undertaken as a bulk process. This bulk process can involve the preparation of up to 1500 transcripts being queried and then printed. It is important during this process that the printer is functional due to the deadline constraints. Once the printing process is completed each student's transcript is placed within a folder ready to be located within their respective graduation date, academic category and time slot. The sorting and finding a transcript is a manual process. In particular finding a transcript or one that has been misplaced can be a time consuming process [9]. This is where the characteristics of RFID can assist the graduation ceremony, as mentioned in Section IV.

To enroll in a academic program a new student will produce an original transcript, attached to their application. At the time of writing this transcript is validated by visual means during the potential enrolment process. There is usually no communication with the external educational facility to validate the transcript presented by the prospective student.

The following Section III-B will present a brief outline of RFID technology and the useful capabilities in the context of this research problem.

\section{B. RFID}

RFID enables the identification of an object utilising radio waves to allow the communication between a reader and a tag. The RFID tag contains a unique identifiable set of numbers and/or characters, which allows the reader to detect and inform an information system. An information system can use this data message to confirm the identity, location, state and time of a object within the confines of a business case. This technology has characteristics that improve the identification of objects compared to other forms such as barcodes [10]. The following section IV will discuss the proposed solution with utilising RFID within the business process of academic transcript generation.

\section{Proposed Solution}

The analysis of this business case is being correlated within the boundary of the business environment of an RFID rationale and deployment methodology. This means the process has to be congruent with the business case study of the organisation and fulfill the expectations of the phase transitional motivators. Examination of academic literature highlights the lack of applied research on how and why an organisation may continue or cease the deployment of an RFID system. In particular [11], [12] describe the lack of publications relating to RFID deployment methodologies and empirically based studies. This research examines the business environment and business case at an organisational level, and why the organisation is contemplating utilising RFID technology.

The proposed solution is to use certain characteristics of RFID technology to enhance the integrity and confidence of the completed transcript. These characteristics include RFID passive tags that are write once, and read multiple times, non line of sight reading and simultaneous identification of multiple tags. The ability to identify multiple transcripts and non line of sight identification, will enhance the production of academic transcripts for graduation. In a particular, it is important to remove a transcript from a designated ceremony, when a student has notified that they cannot attend.

The proposed process is to embed a passive $922 \mathrm{MHz}$ EPC 96 Bit tag within the preprinted paper, that are used to produce academic transcripts. The purpose of this is to enhance the integrity and confidence of the completed transcript. Currently their are businesses selling RFID enabled printers and associated tags, which reduces the complexity of the technology. During the print process the students academic details are printed onto the paper and the embedded RFID tag is encoded. The tag will be coded with an identifier that will be linked to the student number in the student information system. The data to be encoded into the RFID tag will not have student identifiable data. The reason for this is to reduce the likely hood of privacy laws being compromised and increase ease of access to the tag identifier. Unlike [1] the data on the RFID tag will not be encrypted. So a person reading the tag will have to download software [1] to read the tag. In the case of [2], the tags are encrypted so designated readers and software is required to access the data.

At the time of writing, discussions have involved a third party to host a web service. This web service will allow the matching of the tag identifier and student number on the transcript. In this case the university will upload the latest data, in relation to any new transcripts printed. This data will be student number and RFID tag identifier. On the back of the transcript will be information that directs the reader 
to a university web-site with instructions on the verification process. In this case the organisation will read the embedded tag and then enter the student number and RFID tag number into the designated web page. The feedback from the website to the user, will confirm if the two parameter are matched.

It was identified that the process would not significantly change the work flow within the graduation section that produces the transcripts. The administrative staff would merely need to ensure the dedicated printers and paper were being used. The process would require changes to the student administration software to assign and link a RFID tag number back to the student's transcript. The system costing at this stage focuses on software re-development, RFID equipment and only marginal administration costs.

\section{COnClusion And Future Work}

This paper presents a possible RFID deployment within the boundaries of a RFID rationale and deployment methodology. The current process of academic transcript generation is being analysed with the view of including RFID technology within this procedure. At the time of writing, the case study is progressing to the infrastructure environment, which is the second phase of the methodology. This phase will investigate the physics and technical requirements of RFID systems for this business case. It is important to understand how the business case is re-engineered from the context of a use case and how this fulfils the objectives of the business case. Once this is accomplished and the PTMs assigned by the organisation for this phase are congruent, the deployment environment will then be examined.

Since this is a work in progress, several limitations need to be addressed. The research presented, is progressing through a methodology [3],[4] that has three phases, with the first phase results reported. The matching of RFID tag identifier and student number for the transcript through a web service, has potential security and authentication issues that will need further examination. To the best of our knowledge this research has not been examined in literature besides published material on websites. Further research is needed on the durability of transcripts with embedded RFID tags and the potential advantages of RFID enabled transcripts to aid the sorting and organising transcripts for a graduation ceremony. The one partner in this research not covered is the student perception and acceptance of rfid enabled transcripts.

The reputation of a university and student are associated with the accuracy and authenticity of their academic transcript. This transcript is a certified record of a student's studies and can be a crucial element in the successful employment within an organisation. It important at a national and international perspective, that protocols and processes have been put in place, which reduce the likely hood of a falsified document. The proposed process presented in this paper may assist The University of Newcastle, to gain competitive advantage, while improving the legitimacy of their academic transcripts.

\section{ACKNOWLEDGMENT}

The authors would like to thank the reviewers for their invaluable feedback.

\section{REFERENCES}

[1] C. Swedberg, "University of Hyderabad Awards E-Diplomas," Oct. 2007; http://www.rfidjournal.com/article/articleview/3699 17.06.2009.

[2] R. Wessel, "UAE Universities Adopt RFID to Thwart Diploma Forgery," June 2009 http://www.rfidjournal.com/article/articleview/4961 17.06.2009

[3] E. Lutton, G. Skinner and B. Regan, 2008. "RFID rationale and deployment methodology". In Proceedings of the 8th Conference on Applied Computer Science, Venice, Italy, Nov. 2008. pp. 211-216.

[4] E. Lutton, B. Regan and G. Skinner, 2009. "RFID Deployment: Supply Chain Case Study", In RFID and Sensor Networks: Architectures, Protocols, Security and Integrations, Edited by Zhang, Yang, and Chen. to be published by Auerbach Publications, Taylor and Francis, CRC Press, June, 2009. (in press)

[5] M. Munday, T. Edwards and R. Delbridge, 2005. "Understanding innovation in small and medium-sized enterprises: a process manifest”. Technovation Vol 25 (10), pp. 1119-1127.

[6] O. E. Williamson, 1964. The Economics of Discretionary Behavior: Managerial Objectives in a Theory of the Firm. Englewood Cliffs, NJ: Prentice-Hall.

[7] K. Weick, 1995. Sensemaking in Organization, Thousand Oaks, CA: Sage

[8] J. March and H. Simon, 1958. Organizations, New York:Wiley

[9] T. Bodhuin, R. Preziosi and M. Tortorella, 2007. "Supporting document management by using RFID technology". Int. J. Internet Protoc. Technol. Vol. 2, (3/4), Dec. 2007, pp. 165175 .

[10] T. Bodhuin, R. Preziosi and M. Tortorella, "Building an RFID Document Management Service", Innovations in Information Technology, Nov. 2006, pp.1-5

[11] C. Chao, J. Yang, W. Jen, "Determining technology trends and forecasts of RFID by a historical review and bibliometric analysis from 1991 to 2005", Technovation Vol 27, (5), May 2007, pp. 268-279.

[12] E. Ngai, K. Moon, F. Riggins and C. Yi, (2008) "RFID research: An academic literature review (1995-2005) and future research directions". International Journal of Production Economics, Elsevier, Vol. 112(2), April, pp. 510-520.

[13] C.M. Roberts, "Radio frequency identification (RFID)", Computers and Security, Volume 25, Issue 1, February 2006, pp. $18-26$. 